\title{
Theory of Mirror Spectrographs II. General Theory of Focal Surfaces and Slit Curvatures
}

\author{
Klaus D. Mielenz
}

\author{
(July 28, 1964)
}

\begin{abstract}
The focus conditions for Czerny-Turner type, two-mirror spectrographs with spatial beam path are derived. It is shown that, in general, the focal surface of the spectrograph is intermediate between the tangential and sagittal focus. A method is developed to calculate the three-dimensional focal surface, as well as to find the curvature of long monochromator slits.
\end{abstract}

\section{Introduction}

The first to build a mirror spectroscope with antisymmetrical beam path was H. Ebert in 1889 [1]. ${ }^{1}$ He had also suggested substitution of a photographic plate for the eyepiece, but H. Kayser [2] thought this was "entirely impractical," and the design was not carried out as a spectrograph at the time.

In 1930, M. Czerny and A. F. Turner [3] discovered the aberration corrective properties of antisymmetrical two-mirror spectrographs. Their work was realized in the subsequent construction of many twomirror monochromators but had little impact on spectrograph design. Spectrographs, at that time, were mainly prism ones, which are usually better built with lens rather than mirror optics [4].

In 1952, W. G. Fastie [5] reinvented Ebert's onemirror system as a simplified version of the CzernyTurner monochromator. As the trend went to larger and, thus, grating spectrographs, Fastie also suggested the Ebert system for a large plane-grating spectrograph. In this "Ebert-Fastie spectrograph," which was first built in 1955 by R. F. Jarrell [6], Ebert's original in-plane (side-by-side) arrangement of slit, grating, and spectrum was replaced by an off-plane (vertical) arrangement so that a longer spectrum could be obtained.

In 1956, W. Leo [7] further improved the corrective properties of the two-mirror system by using different focal lengths for collimator and camera.

The Czerny-Turner system is still of great practical interest; the one-mirror mounting for its simplicity, and the two-mirror one because of a still better definition of the spectrum.

Yet, theory is still incomplete. Usually, a twodimensional analysis is made, where an horizontal cross section is substituted for the spectrograph itself, and the corresponding focal curve for its actual focal surface. H. Kaiser, F. Rosendahl, and this author [8], and later S. A. Khrshanovskii [9] and O. Vierle [10], have treated Ebert and Ebert-Fastie systems in this manner. Eikonal theories that included two-mirror systems were worked out by K. Kudo [11] and this author [12] but, again, any

${ }_{1}^{1}$ Figures in brackets indicate the literature references at the end of this paper. appreciable deviation from a plane system was not accounted for.

In a two-dimensional analysis, no distinction can be made between in-plane and off-plane systems. It may easily be seen, however, that a significant distinction does exist.

In the in-plane Ebert spectrograph of figure 1a, for instance, slit and spectrum lines extend perpendicular to a tangential plane of the system. The optimal focus of the spectrograph is therefore simply the tangential focus of the mirror.

(a)

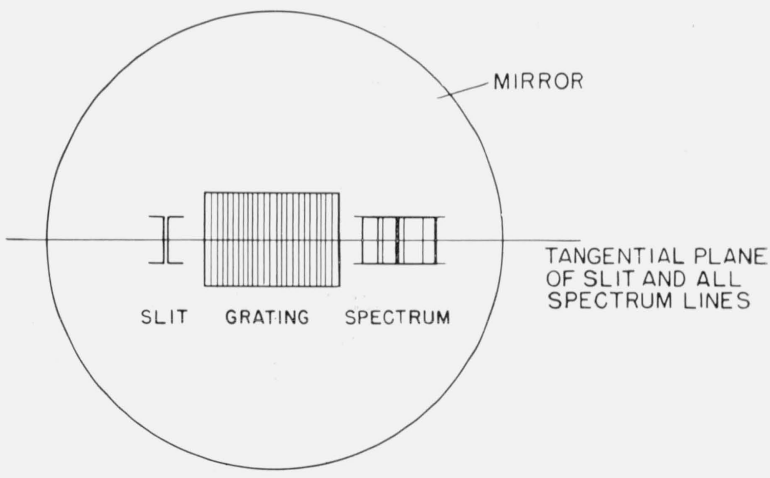

(b)

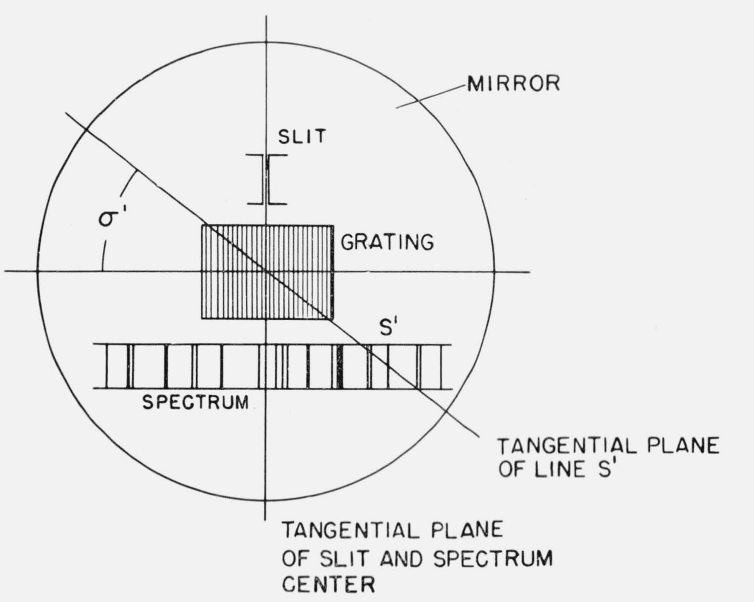

FigURE 1. End-on views of (a) Ebert spectrograph and (b) Ebert-Fastie spectrograph. 
In the off-plane Ebert-Fastie spectrograph of figure 1b, however, a common tangential plane exists only for the slit and the central line of the spectrum. Both lie in this plane and, so, must be at the sagittal foci of the mirror. All other spectrum lines form an angle $\sigma^{\prime}$ with the respective tangential planes of the spectrograph and must therefore be at an intermediate position between the two astigmatic foci, depending upon the value of $\sigma^{\prime}$. For the extreme ends of the spectrum, $\sigma^{\prime}$ approaches zero so that these must be at, or near, the tangential focus of the mirror. Existing theory surmises the focus to be tangential throughout the spectrum.

A two-dimensional analysis will also fail to determine the curvature of monochromator slits, even for in-plane systems. Fastie [5, 13] has considered an additional vertical cross section through the spectrograph to solve the problem, but a threedimensional analysis may be expected to give still more insight.

Thus, a theory is presented in this paper that takes into account the actual solid geometry of the spectrograph. Based upon the most general case of a three-dimensional two-mirror system, it comprises all known types of antisymmetrical mirror spectrographs, with either one or two mirrors, in-plane or off-plane, and with either plane reflectance gratings or Littrow prisms as dispersive elements (though gratings, only, will be explicitly treated in the following). The theory permits calculation of focal surfaces as well as slit curvatures.

An outline of the theory, only, is given. Applications to specific types of spectrographs will be published subsequently.

\section{Basic Geometry of the Spectrograph}

Figure 2 shows the beam path in the spectrograph, where $S$ is a point of the slit and $S^{\prime}$ its image. The principal ray from $S$ to $S^{\prime}$ intersects the grating

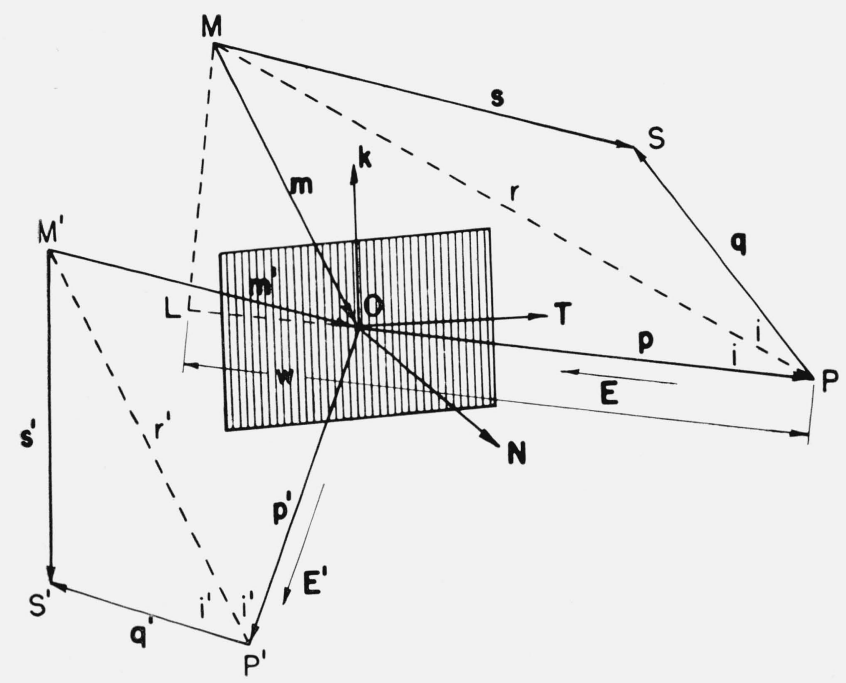

Figure 2. Two-mirror spectrograph. center $O$ and strikes the spherical collimator and camera mirrors at $P$ and $P^{\prime}$, respectively. $M$ and $M^{\prime}$ are the centers of curvature of the two mirrors, $r$ and $r^{\prime}$ their radii.

Let, on the slit side, $\mathbf{s}, \mathbf{m}, \mathbf{p}$, and $\mathbf{q}$ be the vectors $\overrightarrow{M S}, \overrightarrow{M O}, \overrightarrow{O P}$, and $\overrightarrow{P S} ; s, m, p$, and $q$ their absolute values. Write

$$
\mathbf{p}=-p \mathbf{E},
$$

where $\mathbf{E}$ is the unity vector in the direction of the principal ray incident upon the grating.

If, then, $w$ is the distance $L P$, where $L$ is the foot of the perpendicular from $M$ on to $O P$, we have

$$
\begin{gathered}
w=r \cos i=\mathbf{E} \cdot(p \mathbf{E}-\mathbf{m})=p-\mathbf{m} \cdot \mathbf{E}, \\
M L^{2}=r^{2} \sin ^{2} i=[\mathbf{E} \times(p \mathbf{E}-\mathbf{m})]^{2}=(\mathbf{m} \times \mathbf{E})^{2},
\end{gathered}
$$

where $i=\angle S P M=\angle M P O$ is the angle of incidence and reflection at $P$. Hence,

$$
\begin{aligned}
w^{2} & =r^{2}-M L^{2}=r^{2}-(\mathbf{m} \times \mathbf{E})^{2}, \\
p & =w+\mathbf{m} \cdot \mathbf{E} .
\end{aligned}
$$

The directions of incidence and reflection, - $\mathbf{E}$ and $\mathbf{q} / q$, and the normal at $P,(p \mathbf{E}-\mathbf{m}) / r$, obey the law of reflection [14]; i.e.,

$$
\mathbf{q} / q=-\mathbf{E}+2[\mathbf{E} \cdot(p \mathbf{E}-\mathbf{m}) / r](p \mathbf{E}-\mathbf{m}) / r,
$$

or, with (2),

$$
\mathbf{q} / q=-\mathbf{E}+2\left(w / r^{2}\right)(p \mathbf{E}-\mathbf{m}) .
$$

Hence, from figure 2,

$$
\begin{aligned}
\mathbf{s} & =\mathbf{m}-p \mathbf{E}+\mathbf{q} \\
& =-q \mathbf{E}-\left(1-2 q w / r^{2}\right)(p \mathbf{E}-\mathbf{m}) .
\end{aligned}
$$

The corresponding primed expression,

with

$$
\begin{gathered}
\mathbf{s}^{\prime}=q^{\prime} \mathbf{E}^{\prime}+\left(1-2 q^{\prime} w^{\prime} / r^{\prime 2}\right)\left(p^{\prime} \mathbf{E}^{\prime}+\mathbf{m}^{\prime}\right), \\
w^{\prime 2}=r^{\prime 2}-\left(\mathbf{m}^{\prime} \times \mathbf{E}^{\prime}\right)^{2}, \\
p^{\prime}=w^{\prime}-\mathbf{m}^{\prime} \cdot \mathbf{E}^{\prime},
\end{gathered}
$$

is obtained for the point vector, $\mathbf{s}^{\prime}=\overrightarrow{M^{\prime} S^{\prime}}$, of the image point $S^{\prime}$. Here, $p^{\prime}$ and $q^{\prime}$ are the distances $O P^{\prime}$ and $P^{\prime} S^{\prime}, \mathbf{E}^{\prime}$ is the unity vector in the direction of the diffracted principal ray, and $\mathbf{m}^{\prime}$ is the vector $\overrightarrow{M^{\prime} O}$.

Let, finally, $\mathbf{N}, \mathbf{T}$, and $\mathbf{k}$ be mutually orthogonal unity vectors attached to the grating center $O$ so that $\mathbf{N}$ is the grating normal and $\mathbf{T}$ and $\mathbf{k}$ are the tangents perpendicular and parallel to the grooves. $\mathbf{E}$ and $\mathbf{E}^{\prime}$ are then related to each other by the grating equations [15],

$$
\mathbf{T} \cdot\left(\mathbf{E}-\mathbf{E}^{\prime}\right)+\mu \lambda / d=0,
$$




$$
\mathbf{k} \cdot\left(\mathbf{E}-\mathbf{E}^{\prime}\right)=0 \text {, }
$$

where $\mu$ is the spectral order, $\lambda$ the wavelength of the diffracted beam, and $d$ the grating constant.

\section{Focal Distances of Slit and Slit Images}

The focal distances of $S$ and $S^{\prime}, q$ and $q^{\prime}$, are still undetermined in eqs (7) and (8). They may be obtained as follows.

The usual design goal, perfectly collimated light between collimator and camera so that the grating or prism ${ }^{2}$ does not cause aberrations, cannot be accomplished in the mirror spectrograph. The collimating mirror produces an astigmatic pencil of rays which, at best, contains parallel rays in one cross section only. While the aberrations of the grating can no longer be completely eliminated under these circumstances, they will be minimized if parallelism of rays is at least accomplished in the horizontal cross section of the grating [15].

In the mirror spectrograph, this requirement, too, can be met only approximately. The collimating mirror can produce perfectly parallel rays only in a tangential cross section if the slit is arranged at the focal distance

$$
q_{t}=\frac{1}{2} r \cos i=\frac{1}{2} w,
$$

or in a sagittal cross section if the focal distance is

$$
q_{s}=r /(2 \cos i)=r^{2} /(2 w) ;
$$

ef. reference [16] and eq (2), and neither of these will in general coincide with the horizontal cross section of the grating.

There is no intermediate focal distance at which perfectly parallel light is produced in an intermediate cross section. Rays will however $b$ э as nearly parallel as possible in a cross section that encloses an angle $\sigma$ with the tangential plane if the focal distance is

$$
\begin{aligned}
q & =q_{t}+\left(q_{s}-q_{t}\right) \sin ^{2} \sigma \\
& =\frac{1}{2} w+(\mathbf{m} \times \mathbf{E})^{2}\left(\sin ^{2} \sigma\right) /(2 w) ;
\end{aligned}
$$

cf. reference [17] and eq (4). In the spectrograph, the angle $\sigma$ between the tangential plane of the collimating mirror (MOP-plane) and the horizontal cross section of the grating (N, T-plane) is given by

$$
\sin ^{2} \sigma=[\mathbf{k} \times(\mathbf{m} \times \mathbf{E})]^{2} /(\mathbf{m} \times \mathbf{E})^{2},
$$

since $(\mathbf{m} \times \mathbf{E})$ and $\mathbf{k}$ are normal to the two planes.

The focal distance, $q$, at which the slit must be located in order that the aberrations of the grating are minimized is then obtained from (15) and (16) as

$$
q=\frac{1}{2} w+[\mathbf{k} \times(\mathbf{m} \times \mathbf{E})]^{2} /(2 w) .
$$

\footnotetext{
2 Anywhere in this chapter, "prism" may be substituted for "grating" [15].
}

The corresponding primed expression,

$$
q^{\prime}=\frac{1}{2} w^{\prime}+\left[\mathbf{k} \times\left(\mathbf{m}^{\prime} \times \mathbf{E}^{\prime}\right)\right]^{2} /\left(2 w^{\prime}\right),
$$

holds for the focal distance, $q^{\prime}$, of $S^{\prime}$.

\section{Focal Surfaces}

Upon introduction of (17) and (18) into (7) and (8), the focal surfaces of the spectrograph are fully determined. Equations (7) and (8) constitute mutually independent formulas that express the point vectors, $\mathbf{s}$ and $\mathbf{s}^{\prime}$, of a slit point $S$ and its images $S^{\prime}$ as functions of the directions, $\mathbf{E}$ and $\mathbf{E}^{\prime}$, of the principal rays incident upon and diffracted by the grating. Any point on the focal surface, s, of the collimator can therefore be imaged into any point on the focal surface, $\mathbf{s}^{\prime}$, of the camera provided that the grating establishes the necessary relationship between $\mathbf{E}$ and $\mathbf{E}^{\prime}$.

\section{Focal Curves of Spectrographs}

For spectrographs, where long slits are rarely used, it usually suffices to consider a point-by-point imaging of slit centers into line centers.

Thus, let a given point vector $\mathbf{s}_{0}$, i.e., a given principal ray $\mathbf{E}_{0}$ represent the slit center $S_{0}$ on the ícal surface of the collimator mirror. The grating equations, (11) and (12), will then provide the directions, $\mathbf{E}_{0}^{\prime}$, of the diffracted rays and, thus, fully determine the location of the line centers, $S_{0}^{\prime}$, as a curve $\mathbf{s}_{0}^{\prime}$ on the focal surface of the camera mirror.

The full focal surfaces of a spectrograph have to be calculated to such an extent, only, as to establish how slit and photographic plate have to be arranged as tangents to these surfaces so that best possible line definition will be obtained.

\section{Monochromator Slit Curves}

For monochromators with long slits, the above theory is yet incomplete. It is still necessary to find the curves on the two focal surfaces along which e trance and exit slit must extend in order to remain images of each other when the grating is rotated about its vertical axis (direction of $\mathbf{k}$ ) to scan the desired wavelength range.

Let the position of the grating for which the two slits are mirror (zero-order) images of each other be given by

$$
\mathbf{N}=\mathbf{i}, \mathbf{T}=\mathbf{j},
$$

so that $\mathbf{i}, \mathbf{j}$, and $\mathbf{k}$ are base vectors that do not rotate with tha grating. Then, let a curve $\mathbf{s}(\mathbf{E})$ on the focal surface of the collimator mirror represent the entrance slit. The exit slit, as the white-light image, is then given as a curve $\mathbf{s}^{\prime}\left(\mathbf{E}^{\prime}\right)$ on the focal surface of the camera mirror for which the reflection law [14] postulates

$$
\mathbf{E}-\mathbf{E}^{\prime}=2(\mathbf{E} \cdot \mathbf{i}) \mathbf{i} .
$$


If, now, the grating is rotated by an arbitrary angle, so that eqs (19) no longer hold, the same curve $\mathbf{s}^{\prime}$ must become a spectral image of the curve $\mathbf{s}$. The grating equation (11), which because of (20) is now reduced to

$$
2(\mathbf{E} \cdot \mathbf{i})(\mathbf{T} \cdot \mathbf{i})+\mu \lambda / d=0,
$$

must then apply to all slit points and therefore to the slit center as well;

$$
2\left(\mathbf{E}_{0} \cdot \mathbf{i}\right)(\mathbf{T} \cdot \mathbf{i})+\mu \lambda / d=0 .
$$

Hence, as the direction of $\mathbf{T}$ is arbitrary,

$$
\mathbf{E} \cdot \mathbf{i}=\mathbf{E}_{0} \cdot \mathbf{i}
$$

With

$$
\mathbf{E} \cdot \mathbf{k}=\mathbf{E}_{0} \cdot \mathbf{k}+\delta
$$

the third direction cosine of $\mathbf{E}$,

$$
\mathbf{E} \cdot \mathbf{j}=\sqrt{1-(\mathbf{E} \cdot \mathbf{i})^{2}-(\mathbf{E} \cdot \mathbf{k})^{2}},
$$

is also expressed as a function of $\mathbf{E}_{0}$ and $\delta$.

Upon introduction of $(21),(22)$, and (23) into (7) the point vector, $\mathbf{s}$, of an arbitrary point of the entrance slit is obtained as

$$
\mathbf{s}=\mathbf{s}_{0}+\Delta \mathbf{s},
$$

where $\mathbf{s}_{0}$, as before, denotes the slit center and $\Delta \mathbf{s}$ is a function of the coordinates of $\mathbf{s}_{0}$ and the running parameter $\delta$. The shape of the entrance slit is thus fully determined.

From (20) and (21),

$$
\mathbf{E}^{\prime} \cdot \mathbf{i}=-\mathbf{E}_{0} \cdot \mathbf{i},
$$

and from (12) and (22),

$$
\mathbf{E}^{\prime} \cdot \mathbf{k}=\mathbf{E}_{0} \cdot \mathbf{k}+\delta,
$$

whence we may determine the exit slit in a like manner.

The author is indebted to O. N. Stavroudis, NBS, for suggesting the vector notation used throughout this paper, which has resulted in a considerable simplification of all formulas.

\section{References}

[1] H. Ebert, Wiedem. Ann. 38, 489 (1889).

[2] H. Kayser, Handbuch der Spectroscopie, p. 626 (S. Hirzel, Leipzig 1900).

[3] M. Czerny and A. F. Turner, Z. Phys. 61, 792 (1930).

[4] K. D. Mielenz, Optik 16, 500 (1959).

[5] W. G. Fastie, J. Opt. Soc. Am. 42, 641 (1952).

[6] R. F. Jarrell, J. Opt. Soc. Am. 45, 259 (1955).

[7] W. Leo, Z. angew. Phys. 8, 196 (1956).

[8] H. Kaiser, K. D. Mielenz, and F. Rosendahl, Z. Instr. kde. 6\%, 269 (1959).

[9] S. A. Khrshanovskii, Optics and Spectroscopy 9, 207 (1960).

[10] O. Vierle, Dissertation TH Munich, 1962.

[11] K. Kudo, Sci. of Light 9, 1 (1960).

[12] K. D. Mielenz, Optik 19, 510 (1962).

[13] W. G. Fastie, J. Opt. Soc. Am. 42, 647 (1952).

[14] M. Herzberger, in Handbook of Physics, p. 6-22 (McGraw-Hill Book Co., New York, N.Y., 1958).

[15] K. D. Mielenz, J. Res. NBS 68C (Eng. and Instr.) No. 4, Pt. I, 195 (1964).

[16] W. Merte, in Handbuch der Physik, Vol. 18, 81 (J. Springer, Berlin 1927).

[17] C. E. Weatherburn, Differential Geometry of Three Dimensions, Vol. 1, 191 (The University Press, Cambridge 1955).

(Paper 68C4-164) 